\title{
Pseudo-Hermiticity versus PT symmetry: The necessary condition for the reality of the spectrum of a non-Hermitian Hamiltonian
}

Ali Mostafazadeh

Citation: Journal of Mathematical Physics 43, 205 (2002); doi: 10.1063/1.1418246

View online: http://dx.doi.org/10.1063/1.1418246

View Table of Contents: http://aip.scitation.org/toc/jmp/43/1

Published by the American Institute of Physics

\section{Articles you may be interested in}

Pseudo-Hermiticity versus PT-symmetry. II. A complete characterization of non-Hermitian Hamiltonians with a real spectrum

Journal of Mathematical Physics 43, 2814 (2016); 10.1063/1.1461427

Pseudo-Hermiticity versus PT-symmetry III: Equivalence of pseudo-Hermiticity and the presence of antilinear symmetries

Journal of Mathematical Physics 43, 3944 (2002); 10.1063/1.1489072

PT-symmetric quantum mechanics

Journal of Mathematical Physics 40, 2201 (1999); 10.1063/1.532860

Must a Hamiltonian be Hermitian?

American Journal of Physics 71, 1095 (2003); 10.1119/1.1574043

Observation of $P T$ phase transition in a simple mechanical system

American Journal of Physics 81, 173 (2013); 10.1119/1.4789549

Pseudo-Hermiticity and generalized PT- and CPT-symmetries

Journal of Mathematical Physics 44, 974 (2003); 10.1063/1.1539304

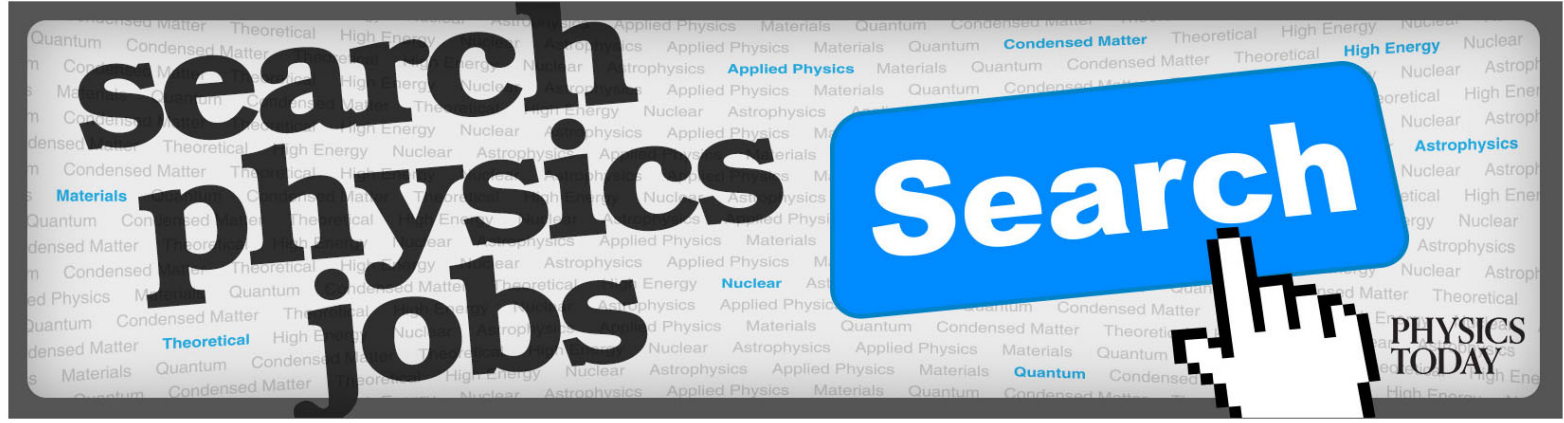




\title{
Pseudo-Hermiticity versus $P T$ symmetry: The necessary condition for the reality of the spectrum of a non-Hermitian Hamiltonian
}

\author{
Ali Mostafazadeha) \\ Department of Mathematics, Koç University, \\ Rumelifeneri Yolu, 80910 Sariyer, Istanbul, Turkey
}

(Received 9 July 2001; accepted for publication 25 September 2001)

\begin{abstract}
We introduce the notion of pseudo-Hermiticity and show that every Hamiltonian with a real spectrum is pseudo-Hermitian. We point out that all the $P T$-symmetric non-Hermitian Hamiltonians studied in the literature belong to the class of pseudoHermitian Hamiltonians, and argue that the basic structure responsible for the particular spectral properties of these Hamiltonians is their pseudo-Hermiticity. We explore the basic properties of general pseudo-Hermitian Hamiltonians, develop pseudosupersymmetric quantum mechanics, and study some concrete examples, namely the Hamiltonian of the two-component Wheeler-DeWitt equation for the FRW-models coupled to a real massive scalar field and a class of pseudo-Hermitian Hamiltonians with a real spectrum. (C) 2002 American Institute of Physics.
\end{abstract}

[DOI: 10.1063/1.1418246]

\section{INTRODUCTION}

The past three years have witnessed a growing interest in non-Hermitian Hamiltonians with real spectra. ${ }^{1-23}$ Based on the results of various numerical studies, Bender and collaborators ${ }^{1,4}$ found certain examples of one-dimensional non-Hermitian Hamiltonians that possessed real spectra. Because these Hamiltonians were invariant under $P T$ transformation, their spectral properties were linked with their $P T$ symmetry. The purpose of this article is to explore the basic structure responsible for the reality of the spectrum of a non-Hermitian Hamiltonian.

By definition, a $P T$-symmetric Hamiltonian $H$ satisfies

$$
\operatorname{PTH}(P T)^{-1}=P T H P T=H,
$$

where $P$ and $T$ are, respectively, the operators of parity and time-reversal transformations. These are defined according to

$$
P x P=-x, \quad P \text { p } P=T p T=-p, \quad T i 1 T=-i 1,
$$

where $x, p$, and 1 are, respectively, the position, momentum, and identity operators acting on the Hilbert space $\mathcal{H}=L^{2}(\mathbb{R})$ and $i:=\sqrt{-1}$. Note that Eq. (2) applies only for the systems whose classical position $x$ and momentum $p$ are real. In this article we shall only be concerned with these systems.

As we mentioned previously, the only reason for relating the concept of $P T$-symmetry and non-Hermitian Hamiltonians with a real spectrum is that most of the known examples of the latter satisfy Eq. (1). Certainly there are Hermitian Hamiltonians with a real spectrum that are not $P T$-symmetric and there are $P T$-symmetric Hamiltonians that do not have a real spectrum. Therefore, $P T$-symmetry is neither a necessary nor a sufficient condition for a Hamiltonian to have a real spectrum. This raises the possibility that the $P T$-symmetry of a Hamiltonian may have

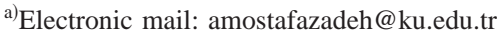


nothing to do with the reality of its spectrum. The interest in $P T$-symmetry seems to be mostly because of the lack of an alternative framework replacing the Hermiticity of the Hamiltonian in ordinary (unitary) quantum mechanics. Much of the published work on the subject concerns the study of various examples and the extension of the concepts developed for Hermitian Hamiltonians to the $P T$-symmetric ones. ${ }^{1-20}$ Recently, Znojil, ${ }^{21}$ Japaridze, ${ }^{22}$ and Kretschmer and Szymanowski ${ }^{23}$ have addressed some of the more fundamental issues regarding the mathematical structure and the interpretation of the $P T$-symmetric quantum mechanics.

Among the common properties of all the $P T$-symmetric Hamiltonians that have so far been studied are the following.

(1) Either the spectrum of the Hamiltonian is real ( $P T$-symmetry is exact) or there are complex-conjugate pairs of complex eigenvalues ( $P T$-symmetry is broken). ${ }^{1,410,12}$

(2) The indefinite inner-product $\langle\langle\mid\rangle\rangle$ defined by

$$
\left\langle\left\langle\psi_{1} \mid \psi_{2}\right\rangle\right\rangle:=\left\langle\psi_{1}|P| \psi_{2}\right\rangle, \quad \forall\left|\psi_{1}\right\rangle,\left|\psi_{2}\right\rangle \in \mathcal{H},
$$

is invariant under the time-translation generated by the Hamiltonian. ${ }^{21,22}$

The main motivation for the present investigation is the remarkable fact that there is no evidence that $P T$-symmetry is the basic structure responsible for these properties. For example, in Ref. 3, the authors construct a class of non- $P T$-symmetric Hamiltonians with a real spectrum. Another example of a non-Hermitian Hamiltonian with similar properties is the Hamiltonian describing the evolution of the solutions of the two-component Wheeler-DeWitt equation for FRW-models coupled with a real massive scalar field. ${ }^{24}$ This Hamiltonian is explicitly "time dependent," "parity-invariant," and non-Hermitian (with respect to the relevant $L^{2}$-norm on the space of two-component wave functions), but the corresponding invariant indefinite inner-product does not involve $P$.

The organization of the article is as follows. In Sec. II, we introduce the concept of a pseudoHermitian operator and derive the basic spectral properties of pseudo-Hermitian Hamiltonians. These coincide with Properties 1 and 2 (with $P$ replaced with a Hermitian invertible linear operator $\eta$ ). In Sec. III, we consider the class of pseudo-Hermitian Hamiltonians that have a complete biorthonormal eigenbasis and show that the pseudo-Hermiticity is a necessary condition for having a real spectrum. In Sec. IV, we explore the pseudo-Hermitian Hamiltonian of the two-component Wheeler-DeWitt equation for FRW-models coupled with a real massive scalar field. In Sec. V, we develop pseudosupersymmetric quantum mechanics. In Sec. VI, we use pseudosupersymmetry to construct a large class of pseudo-Hermitian Hamiltonians with a real spectrum. In Sec. VII, we present our concluding remarks.

\section{PSEUDO-HERMITIAN HAMILTONIANS}

We first give a few definitions. Throughout this paper we will assume that all the inner product spaces are complex. The generalization to real inner product spaces is straightforward.

Definition 1: Let $V_{ \pm}$be two inner product spaces endowed with Hermitian linear automorphisms $\eta_{ \pm}$(invertible operators mapping $V_{ \pm}$to itself and satisfying)

$$
\forall v_{ \pm}, w_{ \pm} \in V_{ \pm}, \quad\left(v_{ \pm}, \eta_{ \pm} w_{ \pm}\right)_{ \pm}=\left(\eta_{ \pm} v_{ \pm}, w_{ \pm}\right)_{ \pm},
$$

where $(,)_{ \pm}$stands for the inner product of $\left.V_{ \pm}\right)$and $O: V_{+} \rightarrow V_{-}$be a linear operator. Then the $\eta_{ \pm}$-pseudo-Hermitian adjoint $O^{\sharp}: V_{-} \rightarrow V_{+}$of $O$ is defined by $O^{\sharp}:=\eta_{+}^{-1} O^{\dagger} \eta_{-}$. In particular, for $V_{ \pm}=V$ and $\eta_{ \pm}=\eta$, the operator $O$ is said to be $\eta$-pseudo-Hermitian if $O^{\sharp}=O$.

Definition 2: Let $V$ be an inner product space. Then a linear operator $O: V \rightarrow V$ is said to be pseudo-Hermitian, if there is a Hermitian linear automorphism $\eta$ such that $O$ is $\eta$-pseudoHermitian. 
Now, consider a quantum system with a possibly non-Hermitian and time-dependent Hamiltonian $H=H(t)$ and a Hilbert space $\mathcal{H}$ which is endowed with a Hermitian linear automorphism $\eta$.

Proposition 1: The Hermitian indefinite inner product $\langle\langle\mid\rangle\rangle_{\eta}$ defined by $\eta$, i.e.,

$$
\left\langle\left\langle\psi_{1} \mid \psi_{2}\right\rangle\right\rangle_{\eta}:=\left\langle\psi_{1}|\eta| \psi_{2}\right\rangle, \quad \forall\left|\psi_{1}\right\rangle,\left|\psi_{2}\right\rangle \in \mathcal{H}
$$

is invariant under the time-translation generated by the Hamiltonian $H$ if and only if $H$ is $\eta$-pseudo-Hermitian.

Proof: First note that the $\eta$-pseudo-Hermiticity of $H$ is equivalent to the condition

$$
H^{\dagger}=\eta H \eta^{-1}
$$

Now, using the Schrödinger equation

$$
i \frac{\mathrm{d}}{\mathrm{d} t}|\psi(t)\rangle=H|\psi(t)\rangle
$$

its adjoint, and Eq. (4), one has for any two evolving state vectors $\left|\psi_{1}(t)\right\rangle$ and $\left|\psi_{2}(t)\right\rangle$ :

$$
i \frac{\mathrm{d}}{\mathrm{d} t}\left\langle\left\langle\psi_{1}(t) \mid \psi_{2}(t)\right\rangle\right\rangle_{\eta}=\left\langle\psi_{1}(t)\left|\left(\eta H-H^{\dagger} \eta\right)\right| \psi_{2}(t)\right\rangle .
$$

Therefore, $\left\langle\left\langle\psi_{1}(t) \mid \psi_{2}(t)\right\rangle\right\rangle_{\eta}$ is a constant if and only if (5) holds.

Note that choosing $\eta=1$ reduces Eq. (5) to the condition of the Hermiticity of the Hamiltonian. Hence pseudo-Hermiticity is a generalization of Hermiticity. Furthermore, observe that a typical $P T$-symmetric Hamiltonian defined on a real phase space $\left((x, p) \in \mathbb{R}^{2}\right)$ has the form $H$ $=p^{2} /(2 m)+V(x)$ where the potential $V(x)=V_{+}(x)+i V_{-}(x)$ has an even real part $V_{+}(x)$ and an odd imaginary part $V_{-}(x)$, i.e., $V_{ \pm}( \pm x)= \pm V_{ \pm}(x)$. It is not difficult to see that such a $P T$-symmetric Hamiltonian satisfies

$$
H^{\dagger}=\frac{p^{2}}{2 m}+V_{+}(x)-i V_{-}(x)=\frac{p^{2}}{2 m}+V_{+}(-x)+i V_{-}(-x)=\text { P H P }=\text { PH } P^{-1} \text {. }
$$

Hence it is $P$-pseudo-Hermitian. In contrast, consider the non-Hermitian Hamiltonians

$$
H_{1}:=p^{2}+x^{2} p, \quad H_{2}:=p^{2}+i\left(x^{2} p+p x^{2}\right) .
$$

Clearly, $H_{1}$ is $P T$ symmetric, but not $P$-pseudo-Hermitian, whereas $H_{2}$ is $P$-pseudo-Hermitian and not $P T$ symmetric. Therefore, $P T$ symmetry and $P$-pseudo-Hermiticity are distinct properties. Note, however, that $H_{1}$ may be pseudo-Hermitian with respect to another Hermitian automorphism $\eta$. We shall explore the relationship between $P T$-symmetry and pseudo-Hermiticity in Sec. III.

The defining condition (5) may also be expressed as the intertwining relation

$$
\eta H=H^{\dagger} \eta
$$

Using this equation together with the eigenvalue equation for the Hamiltonian, namely $H\left|E_{i}\right\rangle$ $=E_{i}\left|E_{i}\right\rangle$, and its adjoint, we can easily show that any two eigenvectors $\left|E_{i}\right\rangle$ and $\left|E_{j}\right\rangle$ of $H$ satisfy

$$
\left(E_{i}^{*}-E_{j}\right)\left\langle\left\langle E_{i} \mid E_{j}\right\rangle\right\rangle_{\eta}=0 .
$$

A direct implication of this equation is the following Proposition.

Proposition 2: An $\eta$-pseudo-Hermitian Hamiltonian has the following properties.

(a) The eigenvectors with a nonreal eigenvalue have vanishing $\eta$-semi-norm, i.e.,

$$
E_{i} \notin \mathrm{R} \quad \text { implies } \quad \|\left|E_{i}\right\rangle \|_{\eta}^{2}:=\left\langle\left\langle E_{i} \mid E_{i}\right\rangle\right\rangle_{\eta}=0 .
$$
i.e.,

(b) Any two eigenvectors are $\eta$-orthogonal unless their eigenvalues are complex conjugates, 


$$
E_{i} \neq E_{j}^{*} \quad \text { implies } \quad\left\langle\left\langle E_{i} \mid E_{j}\right\rangle\right\rangle_{\eta}=0
$$

In particular, the eigenvectors with distinct real eigenvalues are $\eta$-orthogonal.

In the remainder of this section, we list a number of simple but remarkable consequences of pseudo-Hermiticity.

Proposition 3: Let $V$ be an inner product space endowed with a Hermitian linear automorphism $\eta, 1: V \rightarrow V$ denote the identity operator, $O_{1}, O_{2}: V \rightarrow V$ be linear operators, and $z_{1}, z_{2} \in \mathrm{C}$. Then,

(a) $1^{\sharp}=1$;

(b) $\left(O_{1}^{\sharp}\right)^{\#}=O_{1}$;

(c) $\left(z_{1} O_{1}+z_{2} O_{2}\right)^{\#}=z_{1}^{*} O_{1}^{\#}+z_{2}^{*} O_{2}^{\#}$,

where $z_{i}^{*}$ stands for the complex conjugate of $z_{i}$.

Proof: (a) and (b) are trivial consequences of the definition of $\#$ and the Hermiticity of $\eta$. (c) follows from this definition and the linearity of $\eta$ and $\eta^{-1}$ :

$$
\left(z_{1} O_{1}+z_{2} O_{2}\right)^{\#}=\eta^{-1}\left(z_{1} O_{1}+z_{2} O_{2}\right)^{\dagger} \eta=z_{1}^{*} \eta^{-1} O_{1}^{\dagger} \eta+z_{2}^{*} \eta^{-1} O_{2}^{\dagger} \eta=z_{1}^{*} O_{1}^{\#}+z_{2}^{*} O_{2}^{\#} .
$$

Proposition 4: Let $V_{\ell}$, with $\ell \in\{1,2,3\}$, be inner product spaces endowed with Hermitian linear automorphisms $\eta_{\ell}$ and $O_{1}: V_{1} \rightarrow V_{2}$ and $O_{2}: V_{2} \rightarrow V_{3}$ be linear operators. Then $\left(O_{2} O_{1}\right)^{\#}$ $=O_{1}^{\#} O_{2}^{\#}$.

Proof: This relation follows from the following simple calculation:

$$
\left(O_{2} O_{1}\right)^{\#}=\eta_{1}^{-1}\left(O_{2} O_{1}\right)^{\dagger} \eta_{3}=\eta_{1}^{-1} O_{1}^{\dagger} \eta_{2} \eta_{2}^{-1} O_{2}^{\dagger} \eta_{3}=O_{1}^{\sharp} O_{2}^{\#} .
$$

Corollary: Pseudo-Hermitian conjugation $\left(O \rightarrow O^{\sharp}\right)$ is a $*$-operation.

Proof: According to Propositions 3 and 4, \# has all the properties of a *-operation.

Proposition 5: Let $V$ be an inner product space endowed with a Hermitian linear automorphism $\eta, U: V \rightarrow V$ be a unitary operator, and $O: V \rightarrow V$ be a linear operator. Then $\eta_{U}:=U^{\dagger} \eta U$ is a Hermitian linear automorphism, and $O$ is $\eta$-pseudo-Hermitian if and only if $O_{U}:=U^{\dagger} O U$ is $\eta_{U}$-pseudo-Hermitian. In other words, the notion of pseudo-Hermiticity is unitary-invariant.

Proof: First we recall that because $U$ is unitary, $\eta_{U}$ is both Hermitian and invertible. Furthermore, we have

$$
\eta_{U}^{-1} O_{U}^{\dagger} \eta_{U}=U^{\dagger} \eta^{-1} U U^{\dagger} O^{\dagger} U U^{\dagger} \eta U=U^{\dagger}\left(\eta^{-1} O^{\dagger} \eta\right) U
$$

Proposition 6: Let $V$ be an inner product space, $\eta_{1}$ and $\eta_{2}$ be Hermitian linear automorphisms, and $O: V \rightarrow V$ be a linear operator. Then the $\eta_{1}$-pseudo-Hermitian adjoint of $O$ coincides with its $\eta_{2}$-pseudo-Hermitian adjoint if and only if $\eta_{2}^{-1} \eta_{1}$ commutes with $O$.

Proof: This statement holds because $\eta_{1}^{-1} O^{\dagger} \eta_{1}=\eta_{2}^{-1} O^{\dagger} \eta_{2}$ implies $O^{\dagger} \eta_{1} \eta_{2}^{-1}=\eta_{1} \eta_{2}^{-1} O^{\dagger}$. Taking the Hermitian adjoint of this relation yields $\left[O, \eta_{2}^{-1} \eta_{1}\right]=0$.

Corollary: If the Hamiltonian $H$ of a quantum system is pseudo-Hermitian with respect to two different Hermitian linear automorphisms $\eta_{1}$ and $\eta_{2}$ of the Hilbert space, then $\eta_{2}^{-1} \eta_{1}$ is a symmetry of the system. Conversely, let $\eta$ be a Hermitian linear automorphism of the Hilbert space, $G$ be a symmetry group of the system whose elements $g$ are represented by invertible linear operators. Then $\eta g$ is a Hermitian linear automorphism and $H$ is $\eta g$-pseudo-Hermitian provided that $g^{\dagger} \eta g=\eta$.

Proof: This is a direct implication of Proposition 6 and the definition of the symmetry, namely $[g, H]=0$ or equivalently $g^{-1} H g=H .^{25}$ 


\section{PSEUDO-HERMITIAN HAMILTONIANS WITH A COMPLETE BIORTHONORNAL EIGENBASIS}

Let $H$ be an $\eta$-pseudo-Hermitian Hamiltonian with a complete biorthonormal eigenbasis $\left\{\left|\psi_{n}, a\right\rangle,\left|\phi_{n}, a\right\rangle\right\}$ and a discrete spectrum. ${ }^{26}$ Then, by definition,

$$
\begin{gathered}
H\left|\psi_{n}, a\right\rangle=E_{n}\left|\psi_{n}, a\right\rangle, \quad H^{\dagger}\left|\phi_{n}, a\right\rangle=E_{n}^{*}\left|\phi_{n}, a\right\rangle, \\
\left\langle\phi_{m}, b \mid \psi_{n}, a\right\rangle=\delta_{m n} \delta_{a b}, \\
\sum_{n} \sum_{a=1}^{d_{n}}\left|\phi_{n}, a\right\rangle\left\langle\psi_{n}, a\left|=\sum_{n} \sum_{a=1}^{d_{n}}\right| \psi_{n}, a\right\rangle\left\langle\phi_{n}, a\right|=1,
\end{gathered}
$$

where $d_{n}$ is the multiplicity (degree of degeneracy) of the eigenvalue $E_{n}$, and $a$ and $b$ are degeneracy labels.

Proposition 7: Let $H$ be a pseudo-Hermitian Hamiltonian with these properties. Then the nonreal eigenvalues of $H$ come in complex conjugate pairs with the same multiplicity.

Proof: According to Eqs. (5) and (11),

$$
H\left(\eta^{-1}\left|\phi_{n}, a\right\rangle\right)=\eta^{-1} H^{\dagger}\left|\phi_{n}, a\right\rangle=E_{n}^{*}\left(\eta^{-1}\left|\phi_{n}, a\right\rangle\right) .
$$

Because $\eta^{-1}$ is invertible, $\eta^{-1}\left|\phi_{n}, a\right\rangle \neq 0$ is an eigenvector of $H$ with eigenvalue $E_{n}^{*}$. More generally, $\eta^{-1}$ maps the eigensubspace associated with $E_{n}$ to that associated with $E_{n}^{*}$. Again, because $\eta^{-1}$ is invertible, $E_{n}$ and $E_{n}^{*}$ have the same multiplicity.

Next, we use the subscript " 0 " to denote real eigenvalues and the corresponding basis eigenvectors and the subscript " ${ }_{ \pm}$" to denote the complex eigenvalues with \pm imaginary part and the corresponding basis eigenvectors. Then in view of Eqs. (11)-(14), we have

$$
\begin{gathered}
1=\sum_{n_{0}} \sum_{a=1}^{d_{n_{0}}}\left|\psi_{n_{0}}, a\right\rangle\left\langle\phi_{n_{0}}, a\right|+\sum_{n_{+}} \sum_{\alpha=1}^{d_{n_{+}}}\left(\left|\psi_{n_{+}}, \alpha\right\rangle\left\langle\phi_{n_{+}}, \alpha|+| \psi_{n_{-}}, \alpha\right\rangle\left\langle\phi_{n_{-}}, \alpha\right|\right), \\
H=\sum_{n_{0}} \sum_{a=1}^{d_{n_{0}}} E_{n_{0}}\left|\psi_{n_{0}}, a\right\rangle\left\langle\phi_{n_{0}}, a\right|+\sum_{n_{+}} \sum_{\alpha=1}^{d_{n_{+}}}\left(E_{n_{+}}\left|\psi_{n_{+}}, \alpha\right\rangle\left\langle\phi_{n_{+}}, \alpha\left|+E_{n_{+}}^{*}\right| \psi_{n_{-}}, \alpha\right\rangle\left\langle\phi_{n_{-}}, \alpha\right|\right) .
\end{gathered}
$$

Repeating the calculation leading to Eq. (14), we find

$$
\begin{gathered}
\eta^{-1}\left|\phi_{n_{0}}, a\right\rangle=\sum_{b=1}^{d_{n_{0}}} c_{b a}^{\left(n_{0}\right)}\left|\psi_{n_{0}}, b\right\rangle, \quad c_{a b}^{\left(n_{0}\right)}:=\left\langle\phi_{n_{0}}, a\left|\eta^{-1}\right| \phi_{n_{0}}, b\right\rangle, \\
\eta^{-1}\left|\phi_{n_{+}}, \alpha\right\rangle=\sum_{\beta=1}^{d_{n_{+}}} c_{\beta \alpha}^{\left(n_{+}\right)}\left|\psi_{n_{-}}, \beta\right\rangle, \quad c_{\alpha \beta}^{\left(n_{+}\right)}:=\left\langle\phi_{n_{-}}, \alpha\left|\eta^{-1}\right| \phi_{n_{+}}, \beta\right\rangle, \\
\eta^{-1}\left|\phi_{n_{-}}, \alpha\right\rangle=\sum_{\beta=1}^{d_{n_{+}}} c_{\beta \alpha}^{\left(n_{-}\right)}\left|\psi_{n_{+}}, \beta\right\rangle, \quad c_{\alpha \beta}^{\left(n_{-}\right)}:=\left\langle\phi_{n_{+}}, \alpha\left|\eta^{-1}\right| \phi_{n_{-}}, \beta\right\rangle,
\end{gathered}
$$

where $c_{a b}^{\left(n_{0}\right)}$ and $c_{\alpha \beta}^{\left(n_{ \pm}\right)}$are complex coefficients. The latter may be viewed as entries of complex matrices $c^{\left(n_{0}\right)}$ and $c^{\left(n_{ \pm}\right)}$, respectively. Because $\eta$ and consequently $\eta^{-1}$ are Hermitian operators, so are the matrices $c^{\left(n_{0}\right)}$ and $c^{\left(n_{ \pm}\right)}$. In particular, we can make a unitary transformation of the Hilbert space to map the biorthonormal system of eigenbasis vectors of the Hamiltonian to a new system in which these matrices are diagonal. We can further rescale the basis vectors so that $c^{\left(n_{0}\right)}$ 
and $c^{\left(n_{ \pm}\right)}$become identity matrices. In the following we shall assume, without loss of generality, that such a transformation has been performed. Then, Eqs. (17)-(19) take the form

$$
\left|\phi_{n_{0}}, a\right\rangle=\eta\left|\psi_{n_{0}}, a\right\rangle, \quad\left|\phi_{n_{ \pm}}, \alpha\right\rangle=\eta\left|\psi_{n_{\mp}}, \alpha\right\rangle .
$$

In particular, combining this result with Eq. (12), we have the following $\eta$-orthonormalization of the eigenvectors of $H$

$$
\left\langle\left\langle\psi_{n_{0}}, a \mid \psi_{m_{0}}, b\right\rangle\right\rangle_{\eta}=\delta_{n_{0}, m_{0}} \delta_{a b}, \quad\left\langle\left\langle\psi_{n_{ \pm}}, \alpha \mid \psi_{m_{\mp}}, \beta\right\rangle\right\rangle_{\eta}=\delta_{n_{ \pm}, m_{\mp}} \delta_{\alpha \beta} .
$$

Next, we solve Eq. (20) for $\left|\psi_{n_{0}}\right\rangle$ and $\left|\psi_{n_{ \pm}}\right\rangle$and substitute the result in Eq. (15). This leads to an explicit expression for $\eta$ that can be easily inverted to yield $\eta^{-1}$. The result is

$$
\begin{gathered}
\eta=\sum_{n_{0}} \sum_{a=1}^{d_{n_{0}}}\left|\phi_{n_{0}}, a\right\rangle\left\langle\phi_{n_{0}}, a\right|+\sum_{n_{+}} \sum_{\alpha=1}^{d_{n_{+}}}\left(\left|\phi_{n_{-}}, \alpha\right\rangle\left\langle\phi_{n_{+}}, \alpha|+| \phi_{n_{+}}, \alpha\right\rangle\left\langle\phi_{n_{-}}, \alpha\right|\right), \\
\eta^{-1}=\sum_{n_{0}} \sum_{a=1}^{d_{n_{0}}}\left|\psi_{n_{0}}, a\right\rangle\left\langle\psi_{n_{0}}, a\right|+\sum_{n_{+}} \sum_{\alpha=1}^{d_{n_{+}}}\left(\left|\psi_{n_{-}}, \alpha\right\rangle\left\langle\psi_{n_{+}}, \alpha|+| \psi_{n_{+}}, \alpha\right\rangle\left\langle\psi_{n_{-}}, \alpha\right|\right) .
\end{gathered}
$$

One can easily check that the Hamiltonian $H$ and the operators $\eta$ and $\eta^{-1}$ as given by Eqs. (16), (22), and (23) satisfy the $\eta$-pseudo-Hermiticity condition (5).

The above-mentioned analysis provides the following necessary and sufficient condition for pseudo-Hermiticity.

Theorem: Let $H$ be a non-Hermitian Hamiltonian with a discrete spectrum and a complete biorthonormal system of eigenbasis vectors $\left\{\left|\psi_{n}, a\right\rangle,\left|\phi_{n}, a\right\rangle\right\}$. Then $H$ is pseudo-Hermitian if and only if one of the following conditions hold

(1) The spectrum of $H$ is real.

(2) The complex eigenvalues come in complex conjugate pairs and the multiplicity of complex conjugate eigenvalues are the same.

Proof: We have already shown in Proposition 7 that pseudo-Hermiticity of $H$ implies at least one of these conditions. To prove that these conditions are sufficient for the pseudo-Hermiticity of $H$, we use $\left\{\left|\psi_{n}, a\right\rangle,\left|\phi_{n}, a\right\rangle\right\}$ to express $H$ in the form (16) and construct $\eta$ according to Eq. (22). Then, by construction, $H$ and $\eta$ satisfy (5).

This theorem reveals the relevance of the concept of pseudo-Hermiticity to the spectral properties of the $P T$-symmetric Hamiltonians considered in the literature. To the best of our knowledge, an analogue of this theorem that would apply to arbitrary $P T$-symmetric Hamiltonians does not exist. A direct implication of this theorem is the following corollary.

Corollary 1: Every non-Hermitian Hamiltonian with a discrete real spectrum and a complete biorthonormal system of eigenbasis vectors is pseudo-Hermitian.

Note that, in general, a non-Hermitian Hamiltonian may not admit a complete biorthonormal system of eigenvectors. The preceding Theorem and Corollary 1 may not apply for these nonHermitian Hamiltonians.

Corollary 2: Every $P T$-symmetric Hamiltonian with a discrete spectrum and a complete biorthonormal system of eigenbasis vectors is pseudo-Hermitian.

Proof: This statement follows from the above-presented Theorem and fact that the eigenvalues of every $P T$-symmetric Hamiltonian with a complete biorthonormal system of eigenbasis vectors come in complex conjugate pairs. To see this, let $|E\rangle$ be an eigenvector of $H$ with eigenvalue $E$, i.e., $H|E\rangle=E|E\rangle$, and $|E\rangle^{\prime}:=P T|E\rangle$. Then 


$$
H|E\rangle^{\prime}=H(P T)|E\rangle=(P T) H|E\rangle=(P T) E|E\rangle=E^{*}(P T)|E\rangle=E^{*}|E\rangle^{\prime},
$$

where we have made use of the linearlity of $P$ and the antilinearlity of $T$.

\section{PSEUDO-HERMITICITY IN MINISUPERSPACE QUANTUM COSMOLOGY}

The Wheeler-DeWitt equation (with a particularly simple factor ordering prescription) for a Freedman-Robertson-Walker (FRW) model coupled to a massive real scalar field has the form

$$
\left[-\frac{\partial^{2}}{\partial \alpha^{2}}+\frac{\partial^{2}}{\partial \phi^{2}}+\kappa e^{4 \alpha}-m^{2} e^{6 \alpha} \phi^{2}\right] \psi(\alpha, \phi)=0,
$$

where $\alpha=\ln a, a$ is the scale factor, $\phi$ is the scalar field, $m$ is the mass of $\phi$, and $\kappa=-1,0$, or 1 depending on whether the universe is open, flat, or closed. ${ }^{27}$ In the two-component representation developed in Ref. 24, this equation takes the form of the Schrödinger equation: $i \dot{\Psi}=H(\alpha) \Psi$ where a dot stands for a derivative with respect to $\alpha$ and

$$
\begin{gathered}
\Psi=\frac{1}{\sqrt{2}}\left(\begin{array}{c}
\psi+i \dot{\psi} \\
\psi-i \dot{\psi}
\end{array}\right), \quad H=\frac{1}{2}\left(\begin{array}{cc}
1+\mathcal{D} & -1+\mathcal{D} \\
1-\mathcal{D} & -1-\mathcal{D}
\end{array}\right), \\
\mathcal{D}:=-\frac{\partial^{2}}{\partial \phi^{2}}+V(\phi, \alpha), \quad V(\phi, \alpha):=m^{2} e^{6 \alpha} \phi^{2}-\kappa e^{4 \alpha} .
\end{gathered}
$$

As seen from these equations $\mathcal{D} / 2$, up to an unimportant additive scalar, is the Hamiltonian of a "time-dependent" simple harmonic oscillator with unit "mass" and "frequency" $\omega=m e^{3 \alpha}$, where $\alpha$ and $\phi$ play the roles of time $t$ and position $x$, respectively.

It is not difficult to check that the two-component Hamiltonian $H$ is not Hermitian with respect to the $L^{2}$-inner product on the space of two-component state vectors $\Psi$. However, its eigenvalue problem can be solved exactly. ${ }^{24}$ For an open or flat FRW universe $(\kappa=-1,0)$ the eigenvalues of $H$ are real. For a closed FRW model, there is a range of values of $\alpha$ for which all the eigenvalues are real. Outside this range they come in complex conjugate imaginary pairs. This suggests that $H$ is a pseudo-Hermitian Hamiltonian. In fact, we can easily check that $H$ is an $\eta$-pseudo Hermitian Hamiltonian for

$$
\eta=\left(\begin{array}{cc}
1 & 0 \\
0 & -1
\end{array}\right)
$$

The indefinite inner product corresponding to (27) is nothing but the Klein-Gordon inner product that is invariant under the "time-translation" generated by $H$.

\section{PSEUDOSUPERSYMMETRIC QUANTUM MECHANICS}

The application of the ideas of supersymmetric quantum mechanics ${ }^{28}$ in constructing nonHermitian $P T$-symmetric Hamiltonians has been considered in Refs. 3, 7, 13, 17, and 19 and a formulation of $P T$-symmetric supersymmetry has been outlined in Refs. 14 and 20. In this section, we develop a straightforward generalization of supersymmetric quantum mechanics that applies for pseudo-Hermitian Hamiltonians.

Definition 3: Consider a $Z_{2}$-graded quantum system ${ }^{29}$ with the Hilbert space $\mathcal{H}_{+} \oplus \mathcal{H}_{-}$and the involution or grading operator $\tau$ satisfying

$$
\tau=\tau^{\dagger}=\tau^{-1} \quad \text { and } \quad \forall\left|\psi_{ \pm}\right\rangle \in \mathcal{H}_{ \pm}, \quad \tau\left|\psi_{ \pm}\right\rangle= \pm\left|\psi_{ \pm}\right\rangle .
$$


Let $\eta$ be an even Hermitian linear automorphism (i.e., $[\eta, \tau]=0$ ) and suppose that the Hamiltonian $H$ of the system is $\eta$-pseudo-Hermitian. Then $H$ (alternatively the system) is said to have a pseudo-supersymmetry generated by an odd linear operator $\mathcal{Q}$ (i.e., $\{\mathcal{Q}, \tau\}=0$ ) if $H$ and $\mathcal{Q}$ satisfy the pseudosuperalgebra

$$
\mathcal{Q}^{2}=\mathcal{Q}^{\# 2}=0, \quad\left\{\mathcal{Q}, \mathcal{Q}^{\sharp}\right\}=2 H .
$$

A simple realization of pseudosupersymmetry is obtained using the two-component representation of the Hilbert space where the state vectors $|\psi\rangle$ are identified by the column vector $\left(\begin{array}{l}\left|\psi_{+}\right\rangle \\ \psi_{-}\end{array}\right\rangle$ of their components $\left|\psi_{ \pm}\right\rangle$belonging to $\mathcal{H}_{ \pm}$. In this representation, one can satisfy the $\eta$-pseudoHermiticity of the Hamiltonian $H$, [i.e., Eq. (5)] and the pseudosuperalgebra (29) by setting

$$
\begin{aligned}
& \tau=\left(\begin{array}{cc}
1 & 0 \\
0 & -1
\end{array}\right), \quad \eta=\left(\begin{array}{cc}
\eta_{+} & 0 \\
0 & \eta_{-}
\end{array}\right), \\
& \mathcal{Q}=\left(\begin{array}{ll}
0 & 0 \\
D & 0
\end{array}\right), \quad H=\left(\begin{array}{cc}
H_{+} & 0 \\
0 & H_{-}
\end{array}\right),
\end{aligned}
$$

where $\eta_{ \pm}$is a Hermitian linear automorphism of $\mathcal{H}_{ \pm}, D: \mathcal{H}_{+} \rightarrow \mathcal{H}_{-}$is a linear operator, and

$$
H_{+}:=\frac{1}{2} D^{\sharp} D, \quad H_{-}:=\frac{1}{2} D D^{\#} .
$$

Note that, by definition, $\mathcal{Q}^{\sharp}=\eta^{-1} \mathcal{Q}^{\dagger} \eta$,

$$
D^{\sharp}=\eta_{+}^{-1} D^{\dagger} \eta_{-},
$$

and that $H_{ \pm}: \mathcal{H}_{ \pm} \rightarrow \mathcal{H}_{ \pm}$are $\eta_{ \pm}$-pseudo-Hermitian Hamiltonians satisfying the intertwining relations

$$
D H_{+}=H_{-} D, \quad D^{\sharp} H_{-}=H_{+} D^{\sharp} .
$$

As a consequence, $H_{+}$and $H_{-}$are isospectral, $D$ maps the eigenvectors of $H_{+}$to those of $H_{-}$, and $D^{\sharp}$ does the converse, except for those eigenvectors that are eliminated by these operators. More specifically, suppose that $H_{ \pm}$has a complete biorthonormal eigenbasis $\left\{\left|\psi_{n}^{ \pm}, a\right\rangle,\left|\phi_{n}^{ \pm}, a\right\rangle\right\}$ satisfying

$$
H_{ \pm}\left|\psi_{n}^{ \pm}, a\right\rangle=E_{n}^{ \pm}\left|\psi_{n}^{ \pm}, a\right\rangle, \quad H_{ \pm}^{\dagger}\left|\phi_{n}^{ \pm}, a\right\rangle=E_{n}^{ \pm *}\left|\phi_{n}^{ \pm}, a\right\rangle .
$$

Then, $D\left|\psi_{n}^{+}, a\right\rangle$ is either zero in which case $E_{n}^{+}=0$, or it is an eigenvector of $H_{-}$with eigenvalue $E_{n}^{+} ; D^{\sharp}\left|\psi_{n}^{-}, a\right\rangle$ is either zero in which case $E_{n}^{-}=0$, or it is an eigenvector of $H_{+}$with eigenvalue $E_{n}^{-}$. Similarly $D^{\dagger}$ and $D^{\# \dagger}$ relate the eigenvectors $\left|\phi_{n}^{ \pm}, a\right\rangle$ of $H_{ \pm}^{\dagger}$.

An interesting situation arises when one of the automorphisms $\eta_{ \pm}$is trivial, e.g., $\eta_{+}=1$. In this case, $H_{+}$is a Hermitian Hamiltonian with a real spectrum, and pseudosupersymmetry implies that the pseudo-Hermitian Hamiltonian $H_{-}$- which is generally non-Hermitian — must have a real spectrum as well. This is not the only way to generate non-Hermitian Hamiltonians with a real spectrum. In the next section we shall use pseudo-supersymmetry to construct a class of nonHermitian Hamiltonians that have a real spectrum.

\section{A CLASS OF NON-HERMITIAN HAMILTONIANS WITH A REAL SPECTRUM}

Consider the class of pseudosupersymmetric systems corresponding to the choices:

$$
\begin{gathered}
\mathcal{H}_{ \pm}=\mathcal{H}=L^{2}(\mathbb{R}), \quad \eta_{ \pm}= \pm P, \\
D=p+f(x)+i g(x),
\end{gathered}
$$


where $f$ and $g$ are real-valued functions. We can express these functions in the form

$$
f(x)=f_{+}(x)+f_{-}(x), \quad g(x)=g_{-}(x)+g_{+}(x),
$$

where $f_{+}$and $g_{+}$are even functions of $x$, and $f_{-}$and $g_{-}$are odd functions. In view of Eqs. (35)-(37), (33), and (32), we have

$$
\begin{gathered}
D^{\sharp}=p-f_{+}(x)+f_{-}(x)+i\left[g_{+}(x)-g_{-}(x)\right], \\
H_{ \pm}=\frac{1}{2}\left(\left[p+f_{-}(x)\right]^{2}+g_{-}^{\prime}(x) \pm g_{-}^{2}-f_{+}^{2}-i\left[2 g_{-}(x) f_{+}(x) \pm f_{+}^{\prime}(x)\right]+K\right), \\
K:=i\left\{g_{+}(x), p\right\}+g_{+}(x)\left[2 i f_{-}(x)-g_{+}(x)\right],
\end{gathered}
$$

where a prime means a derivative and $\{$,$\} stands for the anticommutator.$

Next, we demand that $H_{+}$is a Hermitian Hamiltonian. The necessary and sufficient condition for the Hermiticity of $H_{+}$and non-Hermiticity of $H_{-}$is

$$
g_{+}(x)=0, \quad g_{-}(x)=-\frac{f_{+}^{\prime}(x)}{2 f_{+}(x)} .
$$

Introducing the even function $\xi(x):=\ln \left|f_{+}(x) / \lambda\right|$ for some $\lambda \in \mathbb{R}-\{0\}$, and using Eqs. (39)-(41), we have

$$
\begin{gathered}
H_{+}=\frac{1}{2}\left(\left[p+f_{-}(x)\right]^{2}+\frac{1}{4} \xi^{\prime}(x)^{2}-\frac{1}{2} \xi^{\prime \prime}(x)-\lambda^{2} e^{2 \xi(x)}\right), \\
H_{-}=\frac{1}{2}\left(\left[p+f_{-}(x)\right]^{2}-\frac{1}{4} \xi^{\prime}(x)^{2}-\frac{1}{2} \xi^{\prime \prime}(x)-\lambda^{2} e^{2 \xi(x)}+2 i \lambda e^{\xi(x)} \xi^{\prime}(x)\right) .
\end{gathered}
$$

By construction, $H_{ \pm}$are pseudo-Hermitian pseudo-supersymmetric partners. In particular, they are isospectral. $H_{+}$happens to be a Hermitian operator. This implies that the eigenvalues of both $H_{+}$ and $H_{-}$are real. Furthermore, for $f_{-}(x) \neq 0, H_{-}$is not $P T$-invariant. This is a concrete example of a non-Hermitian Hamiltonian with a real spectrum that fails to be $P T$-symmetric.

Equation (43) provides a large class of non-Hermitian Hamiltonians with a real spectrum whose members are determined by the choice of functions $f_{-}$and $\xi$. This class includes Hamiltonians with a discrete spectrum. For example let $\xi(x)=-(x / \ell)^{2 n}$, where $n$ is a positive integer and $\ell$ is a positive real parameter with the dimension of length. Then

$$
\begin{gathered}
H_{ \pm}=\frac{1}{2}\left[p+f_{-}(x)\right]^{2}+V_{ \pm}(x), \\
V_{+}=\frac{1}{2}\left(n^{2} \ell^{-4 n} x^{4 n-2}+n(2 n-1) \ell^{-2 n} x^{2 n-2}-\lambda^{2} e^{-2 \ell^{-2 n} x^{2 n}}\right), \\
V_{-}=\frac{1}{2}\left(-n^{2} \ell^{-4 n} x^{4 n-2}+n(2 n-1) \ell^{-2 n} x^{2 n-2}-\lambda^{2} e^{-2 \ell^{-2 n} x^{2 n}}-4 i \lambda n \ell^{-2 n} x^{2 n-1} e^{\left.-\ell^{-2 n} x^{2 n}\right) .}\right.
\end{gathered}
$$

It is not difficult to see that $H_{+}$is a Hermitian Hamiltonian with a discrete spectrum. Therefore, $H_{-}$has a real discrete spectrum as well.

\section{CONCLUSION}

In this article, we have introduced the concept of a pseudo-Hermitian operator and showed that the desirable spectral properties attributed to $P T$-symmetry are in fact consequences of pseudo-Hermiticity of the corresponding Hamiltonians. We have derived various properties of pseudo-Hermitian conjugation and pseudo-Hermitian operators. In particular, we showed how the defining automorphism $\eta$ is linked to the eigenvectors of an $\eta$-pseudo Hermitian Hamiltonian $H$ with a complete biorthonormal eigenbasis. As the corresponding eigenbasis is subject to gauge transformations, the automorphism with respect to which $H$ is pseudo-Hermitian is not unique. This raises the question of the classification of the equivalence classes of automorphisms that lead 
to the same notion of pseudo-Hermiticity for a given Hamiltonian. We have given a brief discussion of this problem and showed its connection with symmetries of the Hamiltonian. We have also developed a generalization of supersymmetry that would apply for general pseudo-Hermitian Hamiltonians, and used it to construct a class of pseudo-Hermitian Hamiltonians with a real spectrum.

A particularly interesting result of our investigations is that all the $P T$-symmetric Hamiltonians that admit a complete biorthonormal eigenbasis are pseudo-Hermitian. In this sense, pseudoHermiticity is a generalization of $P T$-symmetry.

For a $P T$-symmetric Hamiltonian, the exactness of $P T$-symmetry implies the reality of the spectrum. More specifically, if an eigenvector $|E\rangle$ is $P T$-invariant, $P T|E\rangle=|E\rangle$, then the corresponding eigenvalue $E$ is real. A similar condition for a general pseudo-Hermitian Hamiltonian is not known. Pseudo-Hermiticity is only a necessary condition for the reality of the spectrum, not a sufficient condition. In contrast, $P T$-symmetry is neither necessary nor sufficient. The exact $P T$-symmetry is a sufficient condition. But for a given $P T$-symmetric Hamiltonian it is not easy to determine the exactness of $P T$-symmetry without actually solving the corresponding eigenvalue problem.

We hope that the concepts developed in this article provide the material for a more rigorous study of the foundation of pseudounitary quantum mechanics.

\section{ACKNOWLEDGMENTS}

I would like to thank M. Znojil for bringing $P T$-symmetry to my attention. This project was supported by the Young Researcher Award Program (GEBIP) of the Turkish Academy of Sciences.

${ }^{1}$ C. M. Bender and S. Boettcher, Phys. Rev. Lett. 80, 5243 (1998).

${ }^{2}$ F. M. Fernández, R. Guardiola, J. Ros, and M. Znojil, J. Phys. A 31, 10105 (1998).

${ }^{3}$ F. Cannata, G. Junker, and J. Trost, Phys. Lett. A 246, 219 (1998).

${ }^{4}$ C. M. Bender, S. Boettcher, and P. N. Meisenger, J. Math. Phys. 40, 2201 (1999).

${ }^{5}$ C. M. Bender, G. V. Dunne, and P. N. Meisenger, Phys. Lett. A 252, 272 (1999).

${ }^{6}$ C. M. Bender and G. V. Dunne, J. Math. Phys. 40, 4616 (1999).

${ }^{7}$ A. A. Andrianov, M. V. Ioffe, F. Cannata, and J.-P. Dedonder, Int. J. Mod. Phys. A 14, 2675 (1999).

${ }^{8}$ C. M. Bender, S. Boettcher, and V. M. Savage, J. Math. Phys. 41, 6381 (2000).

${ }^{9}$ G. A. Mezincescu, J. Phys. A 33, 4911 (2000).

${ }^{10}$ E. Delabaere and D. T. Trinh, J. Phys. A 33, 8771 (2000).

${ }^{11}$ B. Bagchi and R. Roychoudhury, J. Phys. A 33, L1 (2000)

${ }^{12}$ A. Khare and B. P. Mandal, Phys. Lett. A 272, 53 (2000).

${ }^{13}$ B. Bagchi, F. Cannata, and C. Quesne, Phys. Lett. A 269, 79 (2000).

${ }^{14}$ M. Znojil, F. Cannata, B. Bagchi, and R. Roychoudhury, Phys. Lett. B 483, 284 (2000).

${ }^{15}$ M. Znojil and M. Tater, J. Phys. A 34, 1793 (2001).

${ }^{16}$ C. M. Bender, G. V. Dunne, P. N. Meisenger, and M. Şimşek, Phys. Lett. A 281, 311 (2001).

${ }^{17}$ F. Cannata, M. Ioffe, R. Roychoudhury, and P. Roy, Phys. Lett. A 281, 305 (2001).

${ }^{18}$ C. M. Bender, M. Berry, P. N. Meisenger, V. M. Savage, and M. Şimşek, J. Phys. A 34, L31 (2001).

${ }^{19}$ P. Dorey, C. Dunning, and R. Tateo, LANL Archives: hep-th/0104119.

${ }^{20}$ M. Znojil, LANL Archives: hep-th/0101038.

${ }^{21}$ M. Znojil, LANL Archives: math-ph/0104012.

${ }^{22}$ G. S. Japaridze, LANL Archives: quant-ph/0104077.

${ }^{23}$ R. Kretschmer and L. Szymanowski, LANL Archives: quant-ph/0105054.

${ }^{24}$ A. Mostafazadeh, J. Math. Phys. 39, 4499 (1998).

${ }^{25}$ N. N. Bogolubov, A. A. Logunov, A. I. Oksak, and I. T. Todorov, General Principles of Quantum Field Theory (Kluwer, Dordrecht, 1990).

${ }^{26}$ J. Wong, J. Math. Phys. 8, 2039 (1967); F. H. M. Faisal and J. V. Moloney, J. Phys. B 14, 3603 (1981).

${ }^{27}$ D. N. Page, in Gravitation: A Banff Summer Institute, edited by R. Mann and P. Wesson (World Scientific, Singapore, 1991).

${ }^{28}$ E. Witten, Nucl. Phys. B 202, 253 (1982); L. E. Gendenshtein and I. V. Krive, Sov. Phys. Usp. 28, 645 (1985); F. Cooper, A. Khare, and U. Sukhatme, Phys. Rep. 251, 267 (1995); G. Junker, Supersymmetric Methods in Quantum and Statistical Physics (Springer, Berlin, 1996).

${ }^{29}$ K. Aghababaei Samani and A. Mostafazadeh, Nucl. Phys. B 595, 467 (2001). 